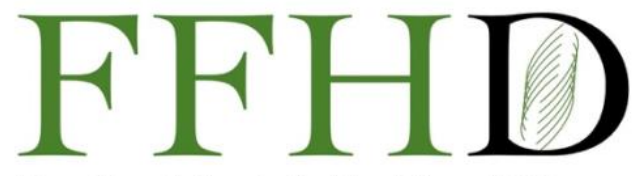

Functional Foods in Health and Disease

\title{
Functional food science: Differences and similarities with food science
}

\author{
Danik Martirosyan ${ }^{1,4^{*}}$, Jessica von Brugger ${ }^{1,2^{*}}$, Sarina Bialow ${ }^{1,3}$
}

${ }^{1}$ Functional Food Center Inc., Dallas, TX, United States; ${ }^{2}$ Loyola University-Chicago, IL, United States; ${ }^{3}$ Syracuse University, Syracuse NY, United States; ${ }^{4}$ Functional Food Institute, San Diego, CA, United States

Corresponding Authors: Jessica von Brugger, Loyola University-Chicago, IL, United States and Danik Martirosyan, PhD, Functional Food Institute, San Diego, CA, United States

Submission Date: August 5 ${ }^{\text {th }}, 2021$; Acceptance Date: August 26 ${ }^{\text {th }}, 2021$; Publication Date: September $2^{\text {nd }}, 2021$

Please cite this as: Martirosyan D., von Brugger J., Bialow S. Functional food science: Differences and similarities with food science. Functional Foods in Health and Disease 2021. 11(9): 408-430. DOI: https://www.doi.org/10.31989/ffhd.v11i9.831

\section{ABSTRACT}

Many nations are facing rising healthcare costs.

The field of functional food science (FFS) has been introduced to combat this. Functional foods are foods with added bioactive compounds which provide a clinically proven health benefit. However, FFS and food science (FS) are often viewed as one and the same. To progress in development and research in the field of FFS, the two must be viewed as separate. Currently, the FDA has not issued or accepted a formal definition for functional foods. In contrast, the FDA accepts and actively

\section{Functional Food Science}

1. Aims to improve health and combat disease

2. Not regulated by FDA

3. Seldom taught in secondary/higher education
4. Many challenges finding evidence to support FFP claim

5. Reliant on quantification for FFP to work as intended

\section{Food Science}

1. Deals with food security,
food preservation, etc.

2. Strictly regulated by FDA

3. Commonly taught in secondary/higher education

$$
\begin{aligned}
& \text { 4. No challenges finding } \\
& \text { evidence to support FFP } \\
& \text { claim }
\end{aligned}
$$

uses FS. This makes it difficult to regulate functional foods and weakens public trust.

The FDA currently has a health claim authorization system in place, but it still fails to properly regulate functional foods. Other countries, such as Japan, have regulatory systems set in place specifically for functional foods. This increases public trust, as there is a strict process that a product has to go through before it is authorized 
for consumption. Countries such as Japan could serve as a model for a functional foods regulatory system in the United States. The Functional Food Center (FFC) has proposed a 15-step system similar to Japan's to authorize functional foods in the U.S. Due to a lack of governmental recognition, there is a large educational gap in secondary schools and higher educational institutions when it comes to FS and FFS. Courses and lessons regarding FS are more available to students than courses and lessons concerning FFS. In addition, the field of FFS faces the challenge of separating its work from the field of FS. Because FFS is concerned with creating functional food products (FFPs) that have a clinically proven health benefits, the scientific research in this field must meet rigorous standards to ensure that the FFP in question has substantial evidence for the health claim. It is important not only to acknowledge the distinction between these two fields, but also to understand the benefit this will have on the well-being of the general population. With FFS as an established field, research can be funded accordingly, and new functional foods can be developed that can prevent or lessen the symptoms of disease. Through regular analysis and measurements of specific biomarkers, FFPs can work alongside western medicine to combat disease and dysfunction. Finally, it is important that a major area of difference is the emphasis on quantity when it comes to FFS. Specific quantities must be outlined and followed in order for FFPs to function as they are intended.

Keywords: Functional Food Science, Food Science, Bioactive Compound, Biomarker, Functional Food Product, Foods for Specific Health Use

CFFC 2021. This is an Open Access article distributed under the terms of the Creative Commons Attribution 4.0 License (http://creativecommons.org/licenses/by/4.0)

\section{INTRODUCTION}

Food is a central component in every person's life. Not only does it contribute to overall health, but it also has generated multiple booming industries. Among these industries are two important branches of science: FS and FFS. Oftentimes, the two are viewed as indistinguishable; however, they must be viewed as separate in order to progress in research and development. FS is defined as "the study of physical, microbial, and chemical makeup of food with applications to develop safe, nutritious, and sustainable foods and innovative packaging" [1]. It has many subsections that focus on different areas in the food and food production industries, including but not limited to: quality control, which focuses on preventing food-borne illnesses; sensory evaluation, which studies how the consumers' senses affect their perception of food; food engineering, which involves the industrial mechanisms used to produce food products; food microbiology, which studies the microorganisms that inhabit food and how they affect the consumer; and foodomics, which focuses on nutrition and health.

Figure 1. shows the different subsections of food science. These subsections help to provide the scientific knowledge to solve the problems that appear in the food and food production systems. FS has many different goals, such as food security, sustainable farming, and food preservation. Food scientists work in all aspects of these systems. They do things like help to develop improved foods, research ways to preserve food better, and produce safe packaging for foods on the market. Food can be defined as an "adequate diet that avoids deficiency" [2]. 


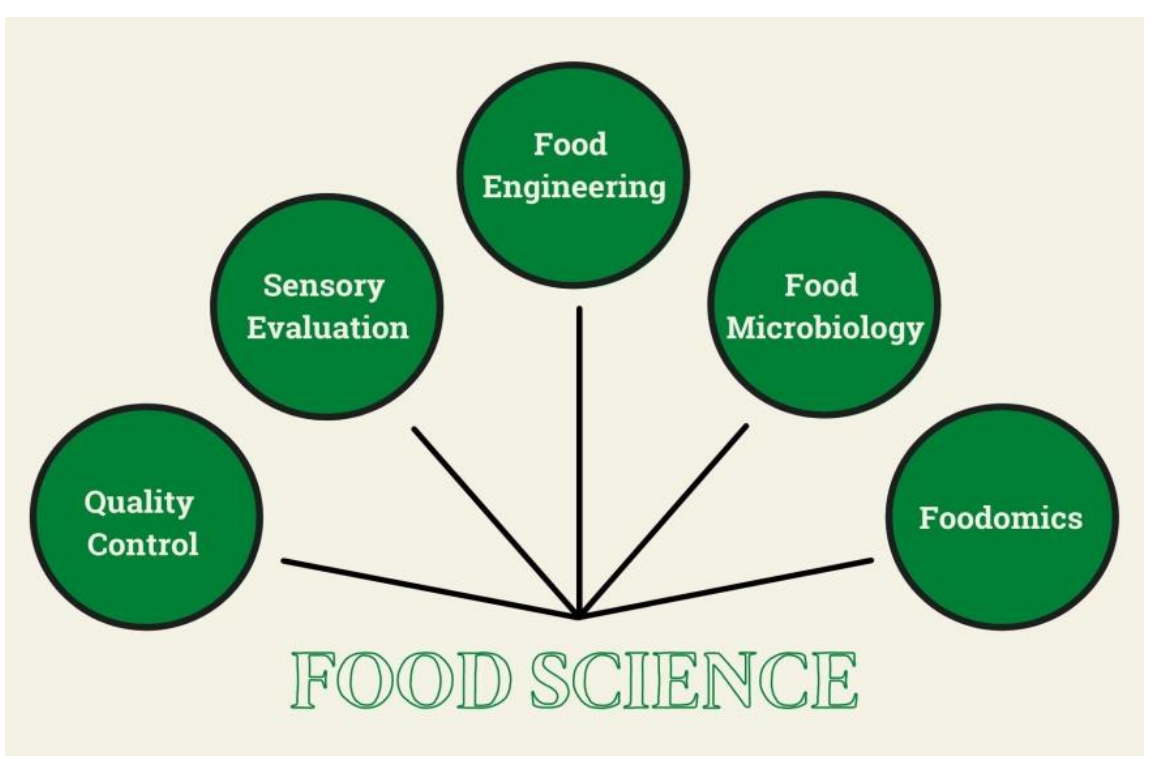

Figure 1. Subsections of FS

Contrarily, FFS is a branch of science that focuses on the nutritive side of foods to be fighters of disease and dysfunction. Functional foods are defined by the Functional Food Center (FFC) as "natural or processed foods that contain biologically-active compounds; which, in defined, effective, non-toxic amounts, provide a clinically proven and documented health benefit utilizing specific biomarkers, to promote optimal health and reduce the risk of chronic/viral diseases and manage their symptoms" [2]. Functional food products (FFPs) are created with two goals. The first goal is to reduce the risk of pathologic processes. The second goal is to improve metabolic and physiologic processes.

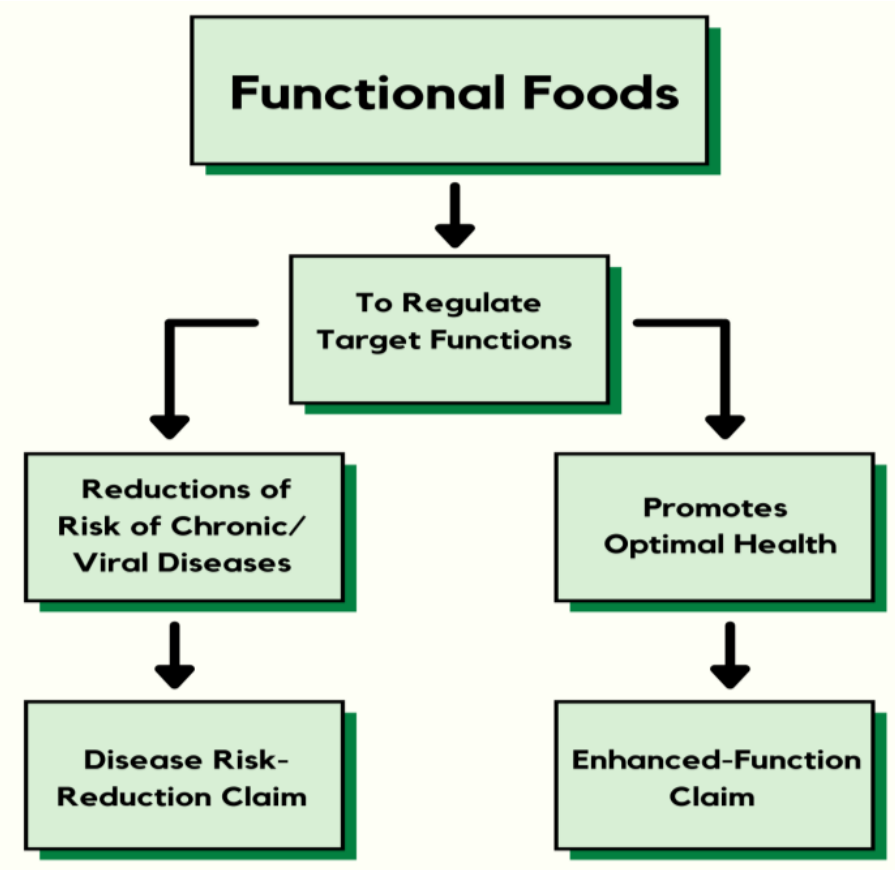

Figure 2. Goals of FFPs [3].

Figure 2. shows the different goals of FFPs. FFPs are not meant to replace western medicine; rather, they are meant to be used alongside western medicine. The FFC has proposed a strict 15-step process to produce an FFP. As FFS is interconnected to many other branches of science, current research 
helps to lay the groundwork. Currently in the United States, functional foods are not officially accepted nor defined by any governmental body, such as the Food and Drug Administration (FDA). On the other end of the spectrum, FS is recognized and actively used by governmental bodies to regulate the United States' food supply. Other countries, such as Japan, have highly developed and regulated systems set in place for the production of FFPs. It is essential that FFPs be defined by a governmental body in the U.S. so that they may be properly regulated. This would allow them to be safely dispersed and would help to gain the public's trust. Additionally, FFS is not taught at nearly the same rate that FS is in secondary schools and higher educational institutions. Public trust should come from governmental regulation that is concerned first and foremost with evaluating scientific evidence regarding FFP claims. Rigorous criteria used to evaluate scientific evidence will ensure that FFPs released to the public are indeed effective and can contribute to a life with improved health and decreased risk of illness. However, the rigorous evaluation that FFS research must face presents the field with challenges not found in the field of FS. Because FS deals with food products that provide a clinically documented health benefit, it must face the challenge of providing substantial evidence to support an FFP claim in order for the FFP in question to be approved by rules and regulations. A notable challenge in FFS research is achieving consistency of results among all published scientific studies of a bioactive compound on health. Each study must be appropriately designed to allow for any results to be biologically relevant to the bioactive compound of interest. Additionally, it must allow for proper statistical analysis to demonstrate significant effects of the bioactive compound on health. Another scientific challenge of FFS is designing studies that are capable of accounting for the numerous interactions between bioactive compounds and other compounds present in the food matrix. Because food is rarely ever composed of a single compound, the consumption of food products leads to potential interactions between compounds that require attention from a scientific perspective. A third challenge that distinguishes the field of FFS from FS relates to the use of valid biomarkers in the research and development of FFPs. Biomarkers provide an opportunity to quantify the effects that a bioactive compound has on health and is therefore a crucial component to any study regarding the development of an FFP. However, some health outcomes and diseases do not have biomarkers that are generally agreed upon. Other health outcomes and diseases have biomarkers that potentially respond in contradictory manners when exposed to the same intervention. These challenges separate the field from FS.

This review explores the similarities and differences between FS and FFS in the United States and the vision of the Functional Food Center in the scientific development of FFPs. Furthermore, it highlights the relevance to the public and the effect that functional foods have on the body by including examples of functional foods that are recommended for consumption.

\section{Functional Food Science vs. Food Science and the} FDA: One major difference seen between FS and FFS is that the FDA officially recognizes FS while it has not officially recognized FFS. An excerpt from the FDA's website says that "the FDA is a science-based public health and regulatory agency responsible for ensuring the safety and proper labeling of foods (including dietary supplements) in the U.S. marketplace" [4]. In order to enforce their laws and regulations, they test for harmful chemicals in foods, ensure that food packaging is safe, and test for allergens and pathogens among other things. In other words, the 
FDA uses FS and its methods to ensure the safety of the US's food supply.

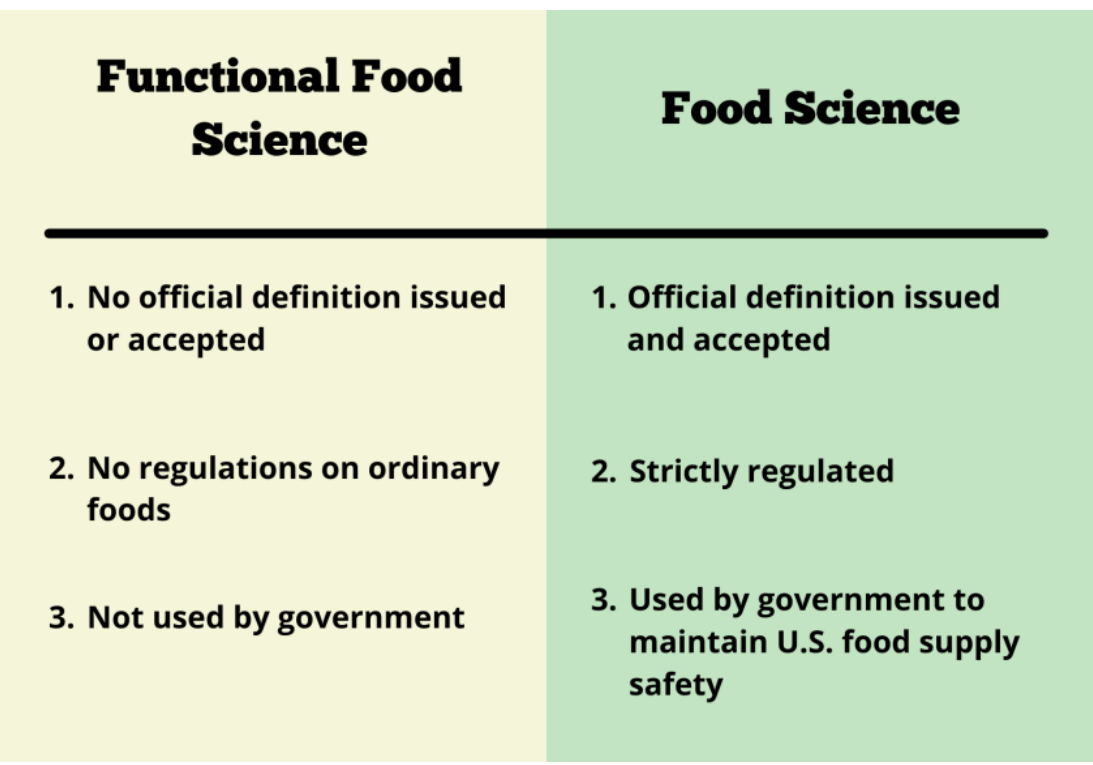

Figure 3. FDA's view on FS vs. FFS.

Figure 3. shows the different views that the FDA has on FS vs. FFS. The FDA has neither accepted nor issued an official definition for functional foods or FFS. This is vital because in order for FFS to progress in research and development, it needs to be recognized by a governmental organization like the FDA. The FDA has also stated that they have no "regulations on the claims for ordinary foodstuffs" [5]. As functional foods are often delivered to the public through "ordinary foodstuffs", this means that functional foods are not regulated. While the FDA has acknowledged certain health claims on food products, they have failed to recognize FFPs as their own category. It is essential that FFPs for the US marketplace be regulated through the US government in order to safely disperse them and gain the public's trust.

FDA's Health Claim Authorization System: One way that the FDA currently regulates food is with the health claim authorization system. A health claim is defined as a statement that characterizes the relationship between a substance and a disease.
These health claims are then evaluated and occasionally authorized by the FDA. In addition, health claims can mention a specific disease/health condition. The health claim must go through a system to be authorized, beginning with a petition sent to the FDA. The claims are then prioritized based on the strength of evidence, whether the claim has previously been reviewed for safety by the FDA, and the impact of the petition on the public. The FDA then collects scientific evidence and data to assess their quality. Additionally, if the petition has not stated dosage recommendation, then the FDA is responsible for determining the proper daily intake of the substance. If the FDA deems the evidence to meet significant scientific agreement, they will authorize the health claim. An example of an authorized health claim is "adequate calcium and vitamin D as part of a healthful diet, along with physical activity, may reduce the risk of osteoporosis in later life" [6]. If all evidence is credible but does not meet their high standards, they issue a "qualified health claim" with a letter of enforcement discretion which specifies what terminology the manufacturer is allowed to put on 
their product. In other words, the manufacturer can still put a health claim on their product if they have a letter of enforcement discretion, but they have to emphasize that this is a qualified health claim and include a disclaimer to prevent misleading the public. An example of a qualified health claim is "scientific evidence suggests, but does not prove, that whole grains (three servings or 48 grams per day), as part of a low saturated fat, low cholesterol diet, may reduce the risk of diabetes mellitus type 2" [6]. If the FDA deems the evidence weak, they will not allow the manufacturer to include any claim, including qualifying language. An example of an authorized health claim in the U.S. is the connection between dietary lipids and cancer. There is strong scientific agreement that a high amount of dietary lipids can increase the risk of cancer. Therefore, manufacturers may make a health claim that foods low in dietary fats may reduce the risk of some types of cancer. However, the health claim authorization system is not foolproof. As previously stated, while the FDA acknowledges certain health claims on food products, they still do not classify them as a functional food. This means that it is difficult to regulate these products. An example of this can be seen with the health claim that soy protein can reduce the risk of coronary heart disease. The FDA is seeking to revoke this health claim as there have been many recent studies that contradict it [7]. The lack of a formal definition or regulation of FFPs in the U.S. restricts future research and development. Manufacturers still need to meet various requirements in order to add a health claim, but they have looser restrictions than other countries who recognize and regulate FFPs. Figure 4. shows different FDA approved health claims and their linked conditions.

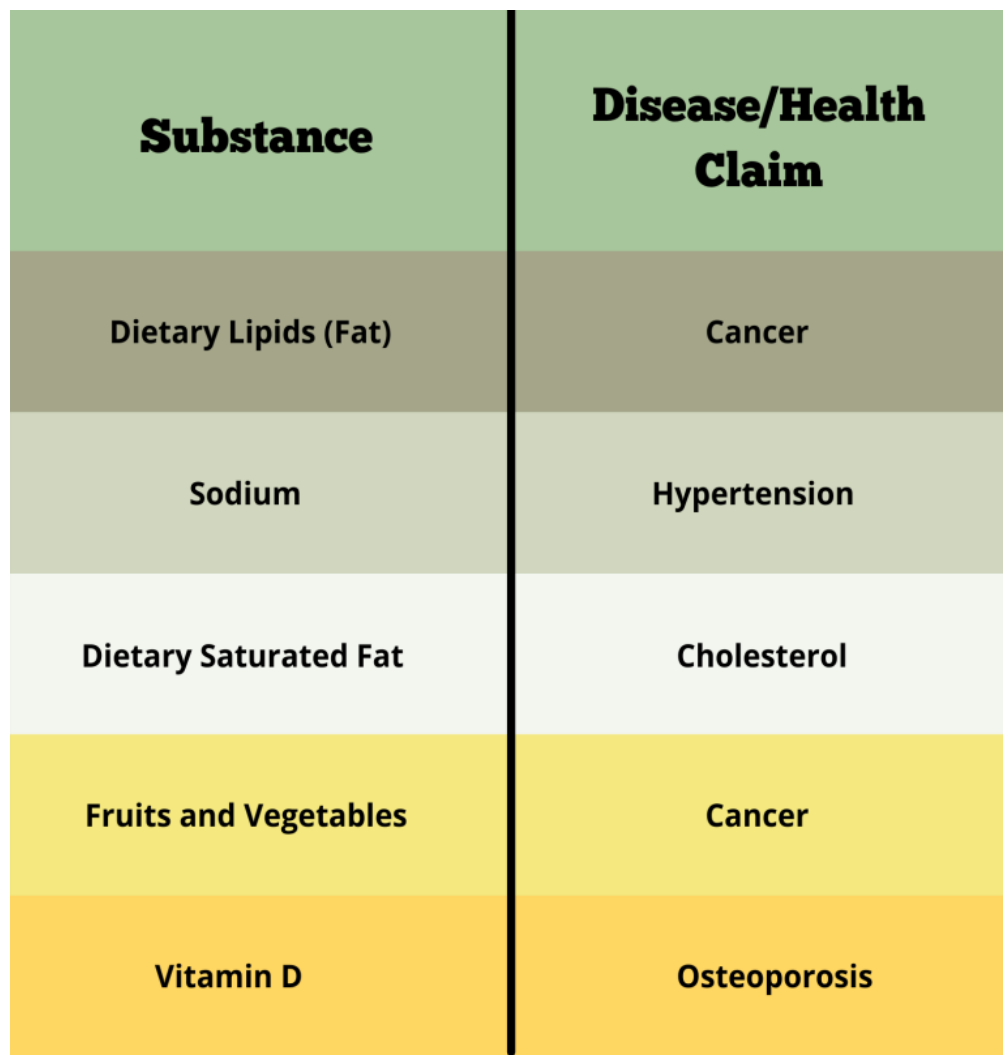

Figure 4. Example of FDA approved health claims and their linked condition [8].

Japan's FOSHU System: For example, in Japan, functional foods have to go through a rigorous process in order to be authorized for public consumption. First, the manufacturer has to submit 
an application to the Consumers Affair Agency. If it meets their standards, the application is passed for evaluation to the Consumers Commission, Food Safety Commission, and Ministry of Health, Labor, and Welfare. If the application passes the standards for these three parties, it is forwarded to the National Institute of Biomedical Innovation, Health, and Nutrition or another registered testing organization so they can validate the analytical process outlined in the product's application. Finally, it is sent back to the Consumers Affair Agency for the final FOSHU approval. They have strict regulations in place for what can and cannot be included on the FFP. For example, no FFP may mention a specific disease name or health condition because the intention of these products is not to cure a disease, but to maintain and/or improve overall health. FOSHU products most commonly have health claims relating to improving gastro-intestinal health by using probiotics [9]. Figure 5. shows the process that a product must go through in order to receive FOSHU approval in Japan.

\section{Applicant}

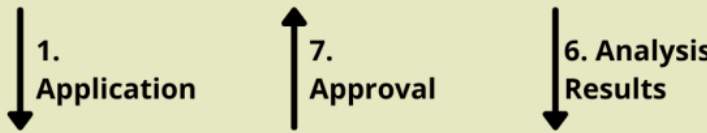

Consumers Affair Agency

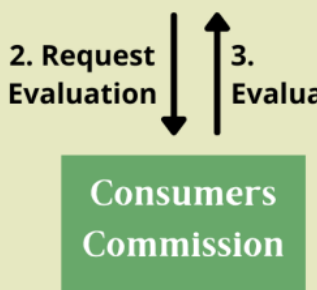

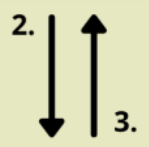

Ministry of

Health, Labor,

$\&$ Welfare

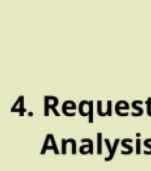

Analysis

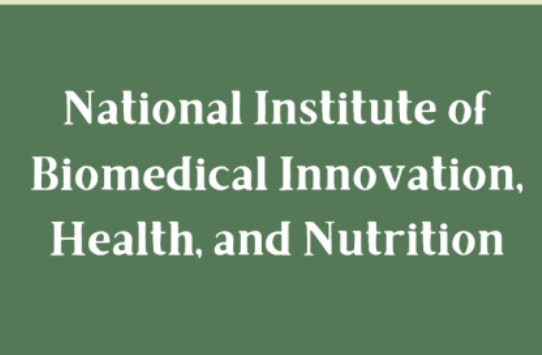

Figure 5. Japan's system to authorize a FOSHU product $[8,10]$.

Additionally, there are five subcategories of FOSHU. The first is the regular FOSHU. Products under this category have an applicable active ingredient and go through a full evaluation process. The second is a standardized FOSHU. These products can only contain active ingredients for which there is adequate scientific evidence proving their efficacy and they must adhere to the daily dosage predetermined by the government. An example of this is Fibersol-2, an ingestible detrin as dietary fiber. This helps regulate $\mathrm{Gl}$ function and blood glucose levels [11]. The third is the risk-reduction FOSHU. So far in Japan, there are only two proven risk reduction compounds: calcium with osteoporosis and folate with neural tube defects. These products also go through a full evaluation process. The fourth is the reauthorized FOSHU, which pertains to products already approved for FOSHU but with changes to the name or flavor. Finally, there are qualified FOSHU products. These products contain active ingredients that may have an unknown mechanism and must include labeling that states that evidence has not necessarily been established and it could possibly exert the health benefit. Additionally, qualified FOSHU products have a special "Qualified Food for Specialized Health Uses" label on them. Clearly, Japan 
has a meticulous system set in place for the authorization of an FFP. Additionally, research shows that products with FOSHU labels have positively influenced consumer's purchasing behavior [12]. This demonstrates that consumers in Japan trust the government's research and their endorsed labeling of FOSHU products.

\section{FFC's Vision for the Research and Development of}

Functional Foods in the U.S.: As previously stated, there is no system set in place to regulate functional foods in the U.S. as no governmental body has officially accepted or defined functional foods. Different organizations hold different definitions for FFPs. This causes FFPs to be misrepresented and leads to public confusion. The FFC has published multiple reviews calling for the improvement of FFPs in the US and consistently vocalizes the need for a new system to be implemented. Ideally, the government and FDA would work alongside the FFC as they have spent years of research and hard work to help develop a proper definition and proposed system to create an FFP. Japan's FOSHU system would serve as a model, and when combined with the FDA's health claim authorization system, would result in a wellgrounded framework for future functional food development.

FFC's Proposed Fifteen Step System to Authorize an FFP: Both FS and FFS work to promote optimal health for their consumers. However, FS focuses on goals such as preservation, food security, packaging, and sustainability. Food scientists work to develop new and better food products, improve the taste of the products for the consumer, and improve processing techniques among other things. On the other hand, FFS aims to prevent and diminish the effects of chronic/viral diseases. They do this by creating an FFP that either reduces the risk of pathological processes or improves the metabolic and physiologic processes.

As previously mentioned, other countries, such as Japan, have formed FFP regulatory systems for public trust and safety. The FFC has proposed a strict 15-step system in order to authorize a functional food for the market in the U.S. In step one, a specific goal is established. An example goal would be to alleviate certain symptoms of a chronic disease. Previous studies are used in order to provide more insight into the goal of the FFP. In step two, extensive research is done in order to determine what bioactive compound should be used in order to achieve the goal of the FFP. Bioactive compounds are primary and secondary metabolites of nutritive and non-nutritive natural components generating health benefits by preventing or managing chronic disease or its symptoms [13]. In step three, a dosage of the bioactive compound is established. This is important because too little or too much of the bioactive compound can nullify the goal. In step four, a biological pathway for the bioactive compound is determined and in step five, a biomarker is chosen. This provides researchers with a measurable variable. This is vital because without a measurable variable, testing cannot be done, and the product cannot be released to the market. In step six, an appropriate food vehicle for the bioactive compound is chosen. In step seven, in vitro and in vivo animal testing is done. This helps to formulate the safest and most effective dosage. In step eight, clinical human trials are performed. This helps to finetune the dosage for human consumption by employing the biomarker. In step nine, a label is created for the FFP. The purpose of this is to properly inform the consumer of things like the dosage recommendations, shelf life, and the most effective way to consume the product. In step ten, the FFP is marketed to the public. This helps educate them about the benefits of the product. In step eleven, epidemiological studies are performed. An epidemiological study is a study that measures the risk of a disease in an exposed population vs. in an unexposed population. This step is crucial because it 
helps to gain the public's trust. However, this step is also a very difficult step as it requires a long period of time and a large subject pool. The FFC is considering issuing different grades of FFPs, such as grade $A$, grade $B$, etc. Because of this, epidemiological studies are preferred, but not $100 \%$ necessary. In step twelve, the data from the epidemiological study is sent to government agencies and third-party organizations for approval. In step thirteen, the FFP is released to the market and in step fourteen, aftermarket research is performed. This aids in monitoring any potential gap between the previous controlled studies and how the FFP affects consumers once it is available on the market. Similar to epidemiological studies, aftermarket research is preferred, but not $100 \%$ necessary. Finally, in the fifteenth step, the FFP is officially established. As you can see, the proposed steps to establish an FFP are very extensive. This is necessary in order to create a safe and trustworthy product for the public to consume, as well as gain FDA approval. Figure 6. shows the 15-step proposed process an FFP must go through in order to be authorized in the US.

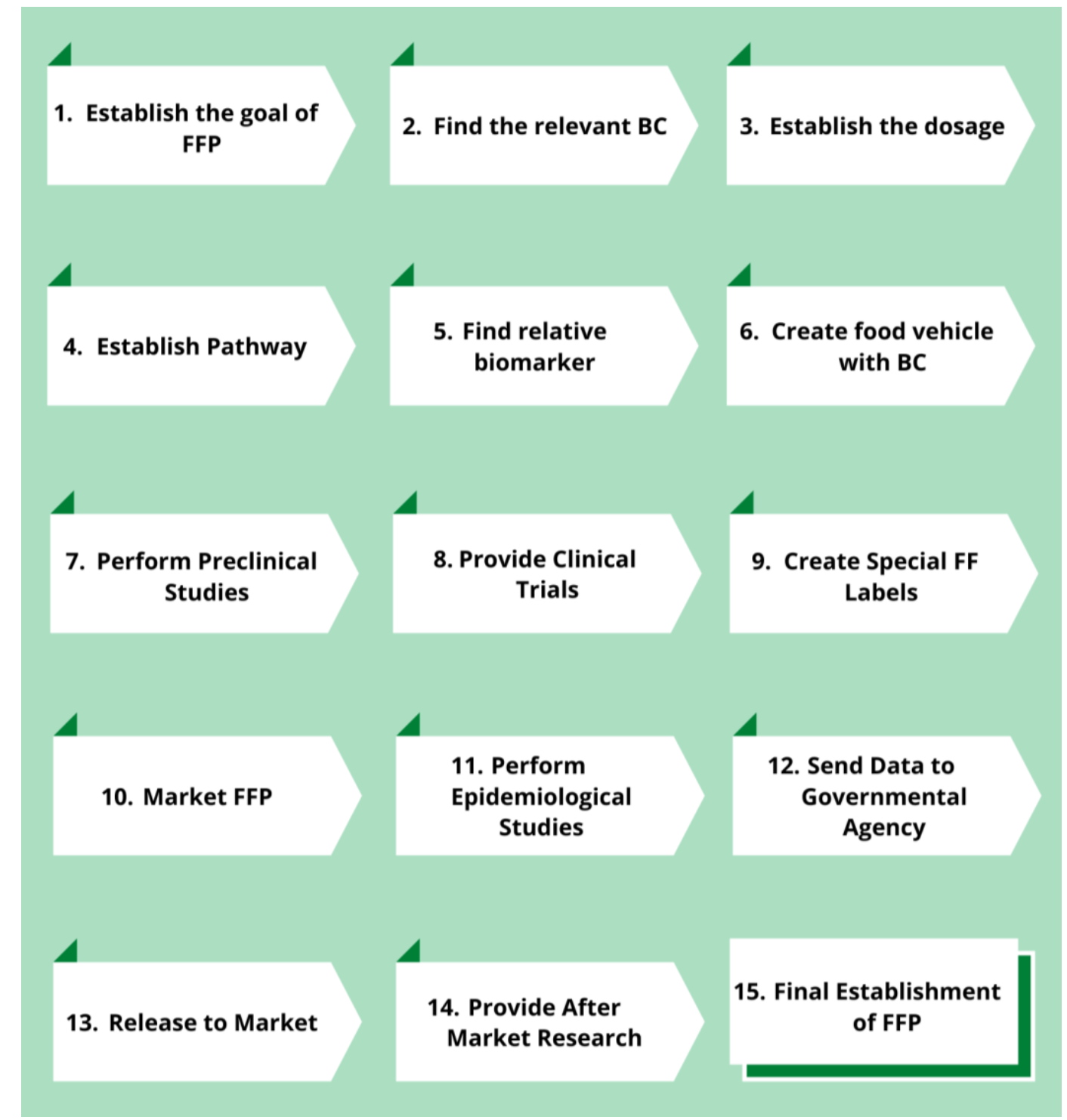

Figure 6. The FFC's proposed system to authorize an FFP [2]. 


\section{METHODOLOGY}

FFS separates itself from other scientific fields of study based on its methodological practices. FFS is reliant on substantial evidence and bioactive compounds in order to consider food products "functional food products". Evidence is evaluated through a detailed 15-step process, which in itself provides insight on the complexity of the science and its determining factors. The foods are analyzed through multiple studies including in vivo studies, in vitro studies, clinical trials and epidemiological studies. It is important to note that each study aims to measure different properties of the potential FFP. With that being said, the variability in data within the studies requires scientists to complete multiple types of studies to get all of the necessary data. Having a wide variety of data gives enough substantial evidence to determine if it qualifies as an FFP. Substantial evidence, measuring the products with specific bioactive compounds and biomarkers, and taking the product through a 15-step process of evaluation is exactly what differentiates FFS from other fields. The attention to detail and processing is imperative to ensure accuracy through and through, which is what FFS hopes to continue in its future endeavors.

Functional Food Science vs. Food Science in Education: Another notable difference between FFS and FS is found in the curriculum available in secondary schools and higher educational institutions. It is common for FS to be taught in a classroom setting. For example, students at Carl Schurz High School in Chicago are being taught FS through a FS Lab. The students are educated on growing fresh food, nutrition, food engineering, and sustainability [14]. Many high schools have either specific classes designed to teach FS, or specialized units in science and health classes. Additionally, many universities have FS classes, FS minors, and FS majors available to their students. According to the U.S. News and World Report, sixty-three universities in the United States offer a FS Major and that number is steadily increasing each year [15]. In contrast, FFS courses and lessons are not taught at nearly the same rate as FS. The FFC as of May of 2021 has 8 published textbooks available to secondary schools and higher educational institutions. Their textbooks range from introductory level books, such as Functional Food Textbook Volume 1: Introduction to Functional Food Science (Third Edition), to specialized areas such as Functional Food Textbook Volume 3: Functional Foods and Cancer. It is also notable that these materials are readily accessible.

One reason that FS is more well known is that FS officially dates back to almost a hundred years ago. FS has always been used throughout history. It is what led to the practices that helped our ancestors farm and domesticate farm animals. Eventually, this led people to develop preservation methods and eventually evolved into the FS we know today. The Institute of Food Technologists (IFT) is an international society of professionals who collaborate to further develop FS. It was created in 1939 and helped popularize the field. FFS, on the other hand, is still relatively new, even internationally. The first time the term "functional food" was even used was in the early ' 80 s in Japan. Japan developed this system in order to help combat the rising healthcare costs the country faced. Because there is only a small number of countries who officially produce and regulate functional foods, there are very few who teach FFS in their school systems. A study was performed in Japan by the International Journal of Clinical Pharmacy to investigate pharmacists' knowledge on functional foods. The results showed a lack of knowledge and training on the topic [16]. Japan has one of the most developed regulatory systems for FFPs and even they struggle to educate medical professionals on this field 
of science. While there has been an increase in the advancement of FFS into secondary and higher educational institutions, it is clear that there is progress to be made.

\section{Scientific and Methodological Challenges in} Functional Food Science: A challenge that distinguishes the field of FFS from FS is that FFS relies on substantiated evidence for the effects of a bioactive compound on health. Substantiated evidence comes from consistent results between multiple types of studies investigating a bioactive compound such as in vitro studies, in vivo studies, clinical intervention studies, and epidemiological studies. The challenge here is having a collective body of evidence that has results that are consistent with one another and indicate that the bioactive component of a functional food has the claimed effect on health. One type of study alone cannot be sufficient to support the health claim of a functional food's bioactive component because each type of previously mentioned study yields valuable, yet limited information on the effects of a bioactive component on health. In vitro studies allow research to determine appropriate amounts of a bioactive compound to administer without putting the life of any living being at risk. In vivo studies in laboratory animals allow research to further refine the amount of the bioactive compound to be administered to minimize any potential adverse health effects in human trials. Trials on humans, especially randomized, double-blinded, placebo-controlled studies, provide the foundation for the body of evidence to support any claim of an FFP and bioactive compound. Yet these studies would not be possible to carry out without preliminary trials in vitro and in vivo in animals because these preliminary studies are necessary to ensure that all human intervention trials are conducted in an ethical manner that does not endanger the lives of human subjects. Figure 7. shows the different studies that contribute to collecting substantiated evidence.

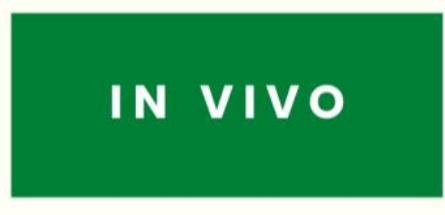

CLINICAL STUDIES

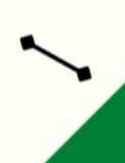

\section{SUBSTANTIATED} EVIDENCE
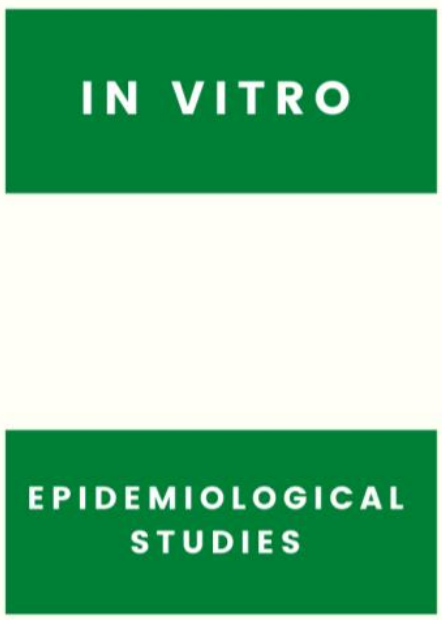

Figure 7. Studies that contribute to substantiated evidence

The process of conducting a human intervention trial that meets the rigorous standards of substantiated scientific evidence under the Process for the Assessment of Scientific Support for Claims on Food (PASSCLAIM) presents another challenge in the field of FFS that is not present in FS. Some PASSCLAIM criteria that may prove challenging for research on FFS include designing a study that accounts for the effect the food matrix and dietary context have on the bioactive component and its functions, ensuring subjects have high compliance with the intake of the food component being studied, and using biomarkers 
that are biologically and methodologically valid to conduct quantitative analysis of the effect of bioactive components on health [17]. The criteria regarding valid biomarkers is especially important to the field of FFS and will be further elaborated on as a challenge that makes FFS distinct from FS later in this section.

The challenge for FFS to meet the criteria of designing a human intervention study that accounts for the influence of the food matrix and dietary context on the effects of the bioactive compound can be observed in previous human intervention studies that delivered unexpected results. Beta-carotene has been extensively studied and was found to have a possible association with the reduction in the risk of lung cancer in epidemiological studies. However, a randomized, double-blinded, placebo-controlled human intervention study using a beta-carotene supplement as an experimental treatment resulted in an increased risk in the incidence of lung cancer for those who took the beta-carotene supplement [18]. This study is a valuable example in showing the necessity that an FFS study be designed to account for the food matrix that is associated with the intake of any bioactive compound. The epidemiological findings found that beta-carotene intake in the form of fruits and vegetables had a risk reduction effect on lung cancer, but a supplement containing isolated beta-carotene had the opposite effect. The behavior of a bioactive compound could differ drastically depending on the form it is consumed in; intake of a bioactive compound in pill form may have a different effect than in liquid or solid food form. When food is consumed, there are multiple interactions occurring between the chemicals present in the food matrix and FFS must account for these interactions in order to develop an effective FFP.

Phytochemicals are a notable subset of bioactive compounds that pose a challenge to FFS research with regards to accounting for the multiple interactions with the food matrix because many of these compounds are found in plants that contain a vast array of phytochemicals that work in concert with the other nutrients and non-nutrients present in the source. Therefore, it is difficult to identify and isolate the most influential bioactive compounds in plants for the purpose of sound, hypothesis-based scientific research. An additional challenge that FFS research faces due to phytochemicals is that environmental conditions will affect the phytochemical profile of a growing plant [19]. Therefore, FFS is challenged with finding a consistent source for phytochemicals for scientific research. If research is conducted with plants of varying phytochemical profiles, results may be inconsistent due to the different levels of certain phytochemicals present in each plant studied and the different levels of interactions between the phytochemicals and other compounds present in a specific plant sample. Any inconsistencies in research that arise from an inconsistent plant source for phytochemicals will hamper the progress of developing FFP support with substantiated evidence.

An additional interaction to consider is between bioactive compounds with potential health benefits and food processing methods. Although a bioactive compound in its natural state may provide health benefits, everyday food processing actions such as applying heat or adding liquid may nullify those healthful properties. For example, isothiocyanates that are found in cruciferous vegetables and released by chewing have been shown to protect against tumor growth [20-21]. 2-phenylethyl isothiocyanate has been recommended in particular to decrease the risk of lung cancer from smoking [20]. However, cooking significantly reduces the uptake of isothiocyanates from cruciferous vegetables because 
the myrosinase enzyme that aids in isothiocyanate absorption is denatured by heat [22-23]

Another scientific challenge that distinguishes FFS is determining the mechanism that explains an FFP's beneficial effect on human health. Finding the mechanism associated with an FFP is reflected in step four of the Functional Food Center's Proposed System to Authorize an FFP [2]. A notable example of this challenge is that scientific research has found a beneficial effect of soy isoflavones on the risk of Cardiovascular Disease. However, the mechanism for this effect has not yet been fully elucidated [24]. Additionally, insufficient research has been done to determine the possible negative consequences to health that soy proteins may bring due to recent research reporting adverse effects of soy protein on lipoprotein(a) and brachial artery flow-mediated dilation [25].

Even when the mechanism of how a bioactive component in a potential FFP is understood, there still may be challenges in implementing that bioactive compound in a manner that is beneficial to human health. Phytoestrogens appear to increase the levels of hormone-binding globulin in the body, which then acts to reduce levels of circulating estrogen and increases the duration of the menstrual cycle by inhibiting gonadotropin output. These factors combined, less estrogen and a longer menstrual cycle, mean that there is lower exposure to estrogen over the course of a life [26-29]. This is notable because less exposure to estrogen over the course of a lifetime is associated with decreased breast cancer risk [28]. However, scientific evidence has also found that supplementing with phytoestrogens may lead to adverse health effects as well. When phytoestrogens were supplemented in soy protein form, subjects had significantly more breast lobular epithelium proliferation [30]. Estrogen from soy protein supplements also leads to an increased amount of hyperplastic cells in aspirated breast fluid from subjects [31]. A study by Allred et al. [32] suggests caution when proceeding to use phytoestrogens as a supplement because this study found that soycontaining diets led to the growth of estrogen dependent tumors in mice. Since scientific research has found that phytoestrogens are involved in a biological mechanism that decreases the risk of breast cancer and that phytoestrogens may lead to an increased risk of cancer, FFS cannot easily proceed with using phytoestrogens as a potential bioactive component in an FFP.

Another example of a challenge in FFS is the use of organosulfur compounds as a bioactive compound in FFPs. Diallyl disulfide and diallyl sulfide in garlic have been found to have anti-carcinogenic effects [33-34]. The mechanism for this effect may be from these bioactive compounds promoting glutathione Stransferase activity in the liver, where the transferase enzyme binds to and detoxifies carcinogens [34]. However, in a study by Fukushima [34] diallyl sulfide promoted liver carcinogenesis, the opposite of the expected inhibition of carcinogenesis. This is another example of how FFS is challenged with conflicting scientific information regarding a bioactive compound's effect on health. If an FFP were to feature organosulfur compounds such as diallyl sulfide, further research must be done to better understand this compound's effects on human health. Similarly, monoterpenes, which are found in the essential oils of fruits like citrus fruits, mint, and herbs, have been found to cause complete regression of mammary carcinomas in a supermajority of rats [35-38]. However, one study found that the monoterpene perillyl alcohol actually had tumor promoting effects [39].

Biomarkers in Functional Food Science: Research in FFS relies on the use of biomarkers because they 
provide a means for quantitatively assessing the effects a bioactive compound has on health. However, certain challenges exist regarding the use of biomarkers in scientific research. Archived biological specimens are a rich source for discovering potential new biomarkers, but the challenge is that there is no standard for the storage and processing of these specimens [40]. The lack of standardization may introduce a source of variability that could impede biomarker discovery because potential confounding variables could obscure any relationships between a candidate biomarker and the value it helps track.

Technological challenges in biomarker discovery also present a challenge to the FFS field that is not present in the FS field. Advanced technology such as quantitative nuclear magnetic resonance spectroscopy (NMR) and liquid chromatographymass spectrometry (LC-MS) are useful tools but have certain limitations. Quantitative NMR methods allow for the identification of new biomarkers with little sample processing necessary. However, this method is not very sensitive to metabolites in small concentrations, so it can only identify the most prevalent metabolites in a given biological sample. LC-MS affords better sensitivity to small amounts of metabolites but requires more extensive workup to process a sample. This workup can be quite expensive when isotopically labeled standards and multipoint calibration curves are used [40]. The challenges presented by the technology involved in the discovery of biomarkers can be viewed as hurdles that biomarker research and FFS research must overcome in order to develop usable biomarkers and FFPs that are supported by scientific evidence.

Biomarker validation is another notable challenge that is present in the biomarker discovery process and by extension, present in FFS. Similar to how FFS research must undergo rigorous evaluation to substantiate any claims on an FFP's effect on health, biomarkers must also undergo rigorous evaluation to be considered suitable for research purposes. No validation process is universally accepted, but all good processes use a systematic approach to validate biomarkers. An exemplary validation process would be the 8-step process by Dragsted et al. that deals with "biomarker plausibility, dose response, time response, robustness, reliability, stability, analytical performance, and reproducibility" [41]. These 8 steps are intended to ensure that biomarkers used in research will help produce results that are accurate and meaningful to the scientific community. However, these criteria that ensure high quality biomarkers also contribute to the slow pace at which valid new biomarkers are discovered. Since research cannot afford to sacrifice quality for speed, the slow pace of new biomarker discovery must be taken as a limitation present in biomarker research and FFS research that depends on biomarkers.

Another challenge in biomarker discovery is that there is simply insufficient data on the fate of chemical compounds after ingestion. Some compounds are metabolically inert and remain identical in composition to the compound found in the food. Others undergo metabolic transformations and become very different from their original, preingested form. A further complication comes from variation of host genetics and microbiome because this variation may lead to differing fates for the same ingested compound. Regarding host genetics, metabolome wide association studies have found that thousands of SNPs and metabolites covary. For the microbiome, a notable example is the conversion of soy isoflavone daidzein to equol in only a subset of people who possess a microbiome with the composition necessary to bring about this conversion [40]. These differing fates of ingested compounds makes the use of biomarkers challenging because a biomarker that is traced well in one individual may 
not be traced well or be entirely absent in another. As a result, scientific research would struggle to reach a sound conclusion regarding the claim of an FFP. Yet another challenge regarding the fate of biomarkers post-ingestion are the various half-lives associated with different biomarkers. Some metabolites like proline betaine in citrus fruits are quickly absorbed and eliminated, leaving a very narrow window to sample the marker after ingestion. Other biomarkers, such as urolithin from microbial metabolism, takes between 30 and 45 hours after ingestion to be detected [40]. Excessively short half-lives and excessively long latency periods of certain biomarkers presents a logistical challenge for biomarker research. The study must be designed in such a way that data is collected at the appropriate time after subjects ingest the bioactive compound via a functional food vehicle.

A human trial study using fecal water cytotoxicity and genotoxicity as biomarkers to study the effect of dairy products in the diet on colon cancer risk serves as an example of the challenges associated with using reliable biomarkers to conduct research. Both cytotoxicity and genotoxicity are generally accepted risk markers for colon cancer. The study found that shifting the subjects from a dairy rich diet to a dairy free one brought about a decrease in cell survival in a cytotoxicity assay but no significant change in genotoxicity from the dietary intervention [42]. Only cytotoxicity significantly changed, yet the other biomarker genotoxicity did not. This raises the question about whether or not the intervention actually brought about the hypothesized effect in the human subjects; one of the two markers indicated a significant change in colon cancer risk, contradicting the other biomarker which indicated no significant change in risk. The fact that cytotoxicity changed significantly but genotoxicity did not as a result of the diet intervention implies that additional work must be done to ensure that biomarkers are well calibrated to track a subject's risk of a disease with regards to dietary intervention. FFS research must be careful to choose the proper biomarker to support the FFP's claim. Additionally, research must consider any possible discrepancies between changes in relevant biomarkers. Possible discrepancies between valid biomarkers are another challenge that FFS must deal with when developing an FFP because such discrepancies would weaken the body of evidence necessary to substantiate an FFP claim.

Relevance to the Public: The necessary distinction between FFS and FS has the capacity to directly benefit the public. It is vital to make steps towards the official recognition of FFS as its own scientific field of study. If successful, proper funding and necessary resources can be put into place. Functional foods, as discovered by FFS, can work in tandem with western medicine to assist with the prevention and/or relief of various diseases. The FFC plays a central role in the establishment of FFS as its own field of study. The FFC hopes to continually strengthen the working definition of FFS in order for it to be recognized by the government and on a global scale. The FFC is directly focused on bettering the wellbeing of the general public and hopes with this establishment to better the lives of those in the United States and globally [1].

\section{Functional Foods Preventing and Relieving Effects of}

Disease: Functional foods have the capacity to prevent and relieve effects of disease. If functional foods are consumed regularly over an extended period of time, they can improve your immune system, making you better equipped to prevent and fight against both viral and chronic diseases. While the definition of functional foods is continuously adapting, the understanding is that functional foods have a clinically proven health benefit as measured by specific biomarkers. These foods are meant to 
promote better health and overall wellbeing, in addition to preventing and relieving effects of disease through the management of associated symptoms [2].

Functional foods play a key role when it comes to strengthening the immune system. Given the scientific advancements made by western medicine, including the availability of vaccines, some may question the need for functional foods as a counterpart to an already effective method for prevention and maintenance. The FFC feels strongly that functional foods can work alongside modern-day medicine. In various situations, functional foods have proven to be especially effective. If a vaccine is not yet available or not effective, functional foods can bridge the gap and decline the rate at which a disease may present itself. If a vaccine is not readily available, as witnessed with COVID-19, consuming functional foods in the meantime will make someone better suited to fight off the disease, as the benefits from these foods will strengthen the immune system.

When discussing functional foods, the elderly, in particular, can see the greatest benefits from regular consumption. While human life expectancy has grown, we see the inverse reaction when looking to the immune system and its decline with age. As a consequence, this puts the older population at greater risk of being immunocompromised and makes them more susceptible to disease. Even if they receive a vaccine, the vaccine may be less effective for them, as their weakened immune system requires a greater dosage than what is given to the average patient. Functional foods can strengthen their ability to fight against viral and chronic diseases and also help relieve symptoms from already existing conditions. With substantial recognition by the government, proper funding of FFS can occur, making it so these discoveries are announced to the general public. More research can occur, leading to the potential to discover even more functional foods, ultimately bettering the immunity of those who need it most [43].

\section{What it Means for Food to be a Functional Food}

Product: Given the positive correlation between the consumption of functional foods and disease prevention, it is important to note specific examples of functional foods and foods advertised as being functional when they are not at all. Most of the time, foods claiming to be functional have no evidence. An FFP can best be authorized by following the 15-step process, as proposed by Functional Food Center. This detailed process ensures that the foods are scientifically proven to have a specific function. The foods must have a recommended dosage, be measured using specific biomarkers, and must be studied through various trials before being released to the public. Even after these foods are marketed, scientists will continue to update information about these foods with aftermarket research [2].

Foods that are often mistaken as functional foods include but are not limited to: foods containing zinc, foods containing omega-3 fatty acids, and foods containing vitamin A. These foods are thought to support the immune system and help fight off viral diseases [43]. While these foods may positively impact the immune system, they cannot be considered "functional foods." Food products like these and others of similar nature tend to lack scientific evidence. For a food to be considered functional, it is best that it follows the 15 -step process 
outlined by the FFC. This ensures the food item is effective and has a clear purpose.

An example of a good candidate for a functional food is Fibersol-2. Fibersol-2, as mentioned previously, is an unflavored powder or liquid additive for foods and drinks. It contains the bioactive compound, digestion-resistant maltodextrin and is multifunctional. It is believed to have positive effects relative to the regulation of healthy glucose levels, thought to increase satiety and assist with intestinal function, and even presumed to help manage metabolic syndrome. It has been analyzed in clinical trials and is perceived to be effective in the prevention of diseases in this realm. While Fibersol-2 has been taken through some of the steps in the 15step process, it still falls short for some of the criteria and needs to be taken through the other steps to confirm that this is a finalized FFP [11].

It is important to educate the public about what makes a food functional. Often, people are misled by marketing tactics that provide misinformation on the label. Confirmed functional foods have great potential for the prevention of disease and the reduction of symptoms. If FFS becomes an established field of its own, misinformation will be retaught correctly. Additionally, customers will be able to purchase foods with the satisfaction that what they are buying is backed by science. Disease can be prevented, symptoms can be managed, and the general population's wellbeing can significantly improve.

Functional Food Science - an Independent Scientific Field of Study: It is imperative to recognize FFS as its own scientific field of study. The discoveries made by these scientists have the capacity to change the way we combat disease as we know it. Doing so would directly benefit the health of the general population and has the capacity to influence the economy through greater sale of food items. From the perspective of safety, recognition is necessary in order to safely carry out all steps of the manufacturing process, even after the products are put on the shelves [2].

The FFC hopes to not only further the scientific discoveries made, but also wants to educate the public on inaccurate information they have been misled to believe all this time. Because the FDA does not require approval for supplements being sold, there is no way of regulating the process. It is important to the FFC that the public understand the true definition of functional foods and the effect supplements have on a person's body [1]. All information is backed by scientific data and measured using specific biomarkers for accuracy. Also, just like the definition of FFS, all foods being sold would be routinely analyzed for accuracy of data, as science is ever-changing.

In addition to ensuring all functional foods that are being marketed are monitored for accuracy, functional foods would be studied alongside others that are beneficial towards the same condition or disease. Scientists will push for manufacturers to design the foods being sold to have a healthy amount of these nutrients all together as one product to maximize benefits [11]. It is crucial to be transparent with the consumer. The public should be educated on the scientific data that has brought that specific item to the shelves. They should also be informed of the benefits that functional food items will have on their health. Instead of listing the vitamins or minerals in that food, it is even more effective to explain what 
this means. The benefits it could have on certain conditions or diseases should be discussed so the consumer is fully aware of what they are getting in this product. There should never be the assumption that someone understands the effects of a certain nutrient and those in FFS should instead be educators on each product label [44]. Figure 8. shows the outcomes of FDA approval in an organized table below.

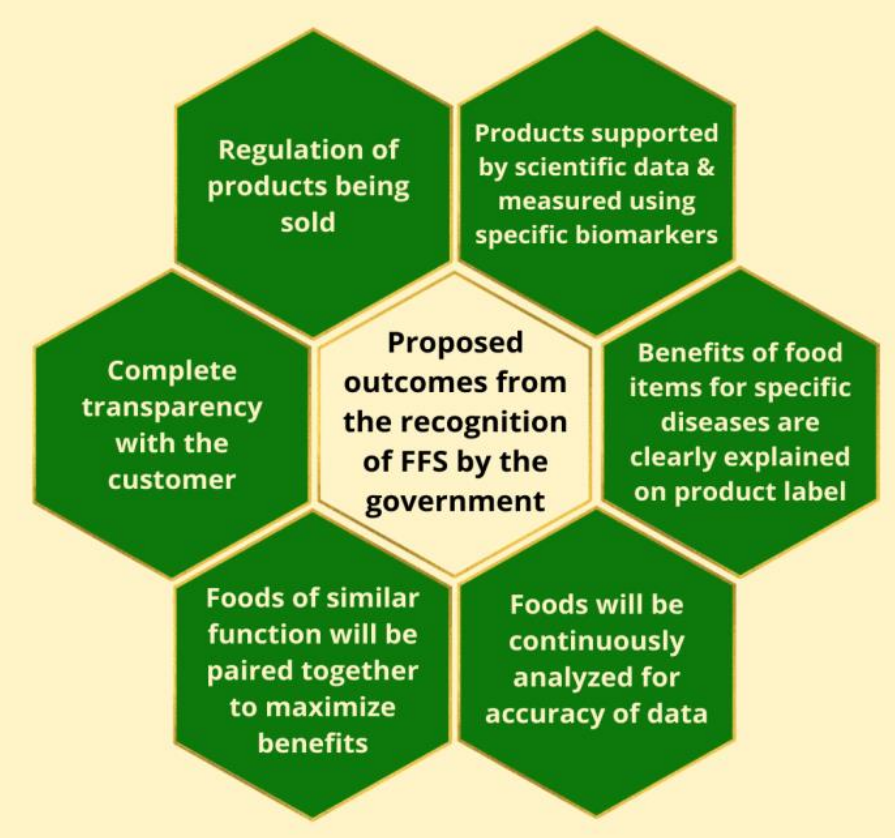

Figure 8. Proposed outcomes from the recognition of FFS by the government [44]. (We have used information from this source to create this image.)

Should FFS become an established field and differentiated from FS, more functional foods can be studied, manufactured, and then given to the public. Throughout this entire process, information to the public will be provided with full transparency and products will be continually monitored even after being put on the shelf. Those who purchase these products will have a clear understanding of what they are buying, in addition to all effects these foods have on their health [1]. The FFC has a key role in this establishment. To officially be recognized by the government and on a global scale, a clear definition of FFS must be established. This, in turn, will pave the way for proper funding and resources to be administered which can further the research being done and ultimately have a positive effect on public health [1].
Quantity in Functional Food Science: One more significant difference between FFS and FS is that FFS revolves around quantification. Examples of this are: the amount of a bioactive compound in an FFP, quantification of the effect of bioactive compounds by using biomarkers, quantification by using special food product labels specifying the amount of a bioactive compound, shelf life, how much of the FFP should be consumed in order to get the health benefit, and what the maximum consumption of the FFP is that you can consume at one time. While FS also relies on quantification, FFS relies on it to a much higher degree. Quantity is vital to FFS because the effects of the bioactive compound, and in turn the FFP, rely on the specific quantities tested in in vivo 
trials, in vitro trials, clinical trials, etc.

FFS as a whole depends greatly on quantity to have its products carry out their intended function. In FFS for an FFP to work as it is intended, it must be taken in a specific, measurable dose. The function of the food item is completely reliant on the amount. Too little of the FFP may cause the item to be ineffective, while too much of the product may cause adverse effects. It is important for the user to follow the outlined dosage recommendation to ensure safe consumption and see the greatest effects from that specific FFP.

\section{DISCUSSION}

Although they are often mistaken for one another, functional food science and food science have many differences. Food science is primarily concerned with food preservation, food security, sustainability, and packaging. On the contrary, functional food science is primarily concerned with developing functional food products that are substantiated by scientific evidence regarding a bioactive compound's effect on health. Currently, the FDA accepts food science while it has not recognized functional food science. The FDA uses a health claim authorization system to add health claims to food products, but this system lacks a formal definition for functional foods. Because functional foods lack their own official category, it is hard for consumers to distinguish normal foods from foods with legitimate functionality. Moreover, food science is a topic that is actively taught in the U.S. education system while there is a lack of education regarding functional food science. Other countries, such as Japan, have a strict regulatory system that could set a precedent for the U.S. The FFC is considering issuing different grades of FFPs based on what steps are met in the proposed 15-step system to authorize a functional food product, such as grade " $A$ ", grade " $B$ ", and grade " $C$ ". For example, a grade " $A$ " functional food product would be a functional food product that completed all 15 steps of the proposed system. A grade " $B$ " functional food product would be a functional food product that went through 14 out of the 15 steps but did not go through an epidemiological study or aftermarket research. A grade " $C$ " functional food product would be a functional food product that completed 13 out of 15 steps of the proposed system but did not go through an epidemiological study and aftermarket research. Other steps, such as clinical trials, are vital to understanding a functional food product's effect, so these steps will not be omitted. Epidemiological studies and aftermarket research are preferred, but not $100 \%$ necessary in order to create a safe functional food product that can be released to the general public.

In addition to this, many challenges arise during the process of gathering scientific evidence to support a functional food product with regards to conducting high quality scientific research. One significant challenge is achieving a general consensus on the effects that a bioactive compound has on health. Because there are various types of scientific studies, each study may produce different results. Another scientific challenge is implementing appropriate study design to allow research to accurately determine the effects of a bioactive compound on health. Functional food science studies must take into account the potential interactions a bioactive compound will have with its dietary context and also use valid biomarkers to quantitatively assess the impact of an FFP with a bioactive compound on general health and disease. The use of valid biomarkers is a challenge in its own sense. Biomarker discovery contains its own set of challenges, such as dealing with the rigorous standards of developing a new valid biomarker. Additional challenges in the 
biomarker field include insufficient understanding of biomarkers with respect to variation among individuals, and possible reporting discrepancies in health changes among biomarkers. Because the implementation of high-quality biomarkers is crucial to provide scientific evidence in support of functional food products, the challenges present in the field of biomarker research extend into the field of functional food science.

Regarding regulation set by the FDA, curricula availability, and scientific challenge, these aspects help differentiate functional food science from food science. By differentiating the two fields of science, functional food science can progress in regulation and legislation. There are not many other articles published thus far that compare functional food science with food science, and also discuss the relevance to the general public. This work can be used to further research about the field and strengthen arguments for the future of functional food science. The conclusions started within this article present an opportunity for greater research to be conducted in the future.

Looking at the methodology, functional food science is unique in its attention to detail and extensive process for certifying a functional food product. While other sciences rely on evidence, functional food science goes a step further and only uses evidence considered substantial. In addition, certain amounts of bioactive compounds are necessary for a food item to be considered a functional food product. While following a 15-step process for certification, a food item will be evaluated in various trials which aim to gather information on different qualities of the food. These trials include in vivo studies, in vitro studies, clinical trials, and epidemiological studies, which all look at different elements of the food item. The data from all trials is combined and analyzed together instead of just relying on one trial. The process intends to rule out food items that do not qualify as functional food products and helps to gather thorough data. This ensures an accurate process that can be utilized to evaluate even more functional foods that have yet to be discovered. Finally looking at quantification, the field of functional food science is dependent on specific numbers for functional food products to be able to carry out their intended function. Functional food science requires specific quantities for safe and proper consumption of their food items. All areas of the field require attention to numbers. Bioactive compounds are measured to deem a product functional, the products are studied in specific trials that measure amounts and in order for safety throughout the process, and the safe amount for shelf life and consumption also are requirements for the product. Functional food science would be nothing without attention to numbers.

\section{CONCLUSION}

Functional food science has the potential to improve the general population's health across the world. The Functional Food Center is considering implementing a grading system for different levels of functional food products. This is similar to Japan's system. They have FOSHU, where, in order to be authorized, products have to go through a strict process. They also have a Foods with Functions Claims system that allows function claims to be added to a product without government evaluation, instead only requiring a clinical trial or a literature review on the potential functional food. If a grading system is implemented for functional food products in the US, this could make them more widely accessible to the general population. 
It is key to recognize the discrepancies between food and functional food products and the differences between functional food science and food science. Food is meant to provide energy for the body and support basic function. However, foods do not require regulatory processes or a process for certification like functional foods do. Functional foods contain biologically active compounds, which in defined, effective, non-toxic amounts have a health benefit. They are measured by specific biomarkers and are meant to improve health by reducing risk of disease and helping to manage symptoms.

Similarly, functional food science is separate from food science. Functional food science describes the preparation of functional foods where foods are used for health purposes. Food science is an entirely different concept which pertains to the physical, microbial and chemical composition of food. Food science is used to identify the nutritional makeup of foods and can play a role in sustainability, food safety, and food packaging.

There is also a need for quantification in the field of functional food science. For food products to be consumed, they must have a specified amount on the label for the user to follow. This ensures the product will work as it is intended and will be used safely. It is important we develop a clear distinction between these concepts, as this is a necessary step to recognize functional food science as its own official field. Articles written in the future should focus on the

\section{REFERENCES}

1. Martirosyan, D., Liufu, J. FFC's Advancement of the Establishment of FFS. Functional Foods in Health and Disease 2020. 10(8), 344-356. DOI: https://doi.org/10.31989/ffhd.v10i8.729

2. Martirosyan, D., Kanya, H. Nadalet, C. Can functional foods reduce the risk of disease? Advancement of functional food definition and steps to create functional food products. Functional Foods in Health and Disease 2021. 11(5), 213-221. DOI: possible classification of functional food products, which helps the field of functional food science. This can help to more clearly define functional food science as a field and can better guide the process of distinguishing functional food products. Eventually, we can establish a single definition across the U.S. and in turn, continue the discovery of more functional foods which can benefit the general public.

Abbreviations: FDA: U.S. Food and Drug Administration, FFC: Functional Food Center, FFP: functional food product(s), FOSHU: Foods for Specified Health Uses, GI: gastrointestinal tract, IFT: Institute of Food technologists, FFS: Functional Food Science, FS: Food Science

Acknowledgements: We would like to thank Harper Lay, an undergraduate student from University of Texas at Austin. With skills in marketing and graphic design, and general understanding of functional food science, she has worked to effectively create graphics and visuals for this article. Additionally, we would like to thank Yallen Bai, an undergraduate student at Cornell University in Ithaca, NY for his help with researching biomarkers.

No external funding was required for this review article.

Conflict of Interest: The authors have no financial interests or conflicts of interest.

https://doi.org/10.31989/ffhd.v11i5.788

3. Roberfroid MB: Concepts and strategy of FFS: the European perspective, The American Journal of Clinical Nutrition. 2000, 71(6), 1660S-1664S. DOI: https://doi.org/10.1093/ajcn/71.6.1660S

4. U.S. Food and Drug Administration (FDA) [https://www.fda.gov/about-fda/what-we-do] Retrieved July 25, 2021.

5. Kwak N, Jukes, DJ. Functional foods. Part 1: the development of a regulatory concept. Food Control 2001, 12(2), 99-107. DOI: https://doi.org/10.1016/S09567135(00)00028-1

6. U.S. Food and Drug Administration (FDA) 
[https://www.fda.gov/food/food-labelingnutrition/questions-and-answers-health-claims-foodlabeling] Retrieved July 25, 2021.

7. Petersen KS: The Dilemma With the Soy Protein Health Claim. Journal of the American Heart Association. 2019,8(13). DOI: https://doi.org/10.1161/jaha.119.013202

8. Sadohara, R., Martirosyan, D. Functional Food Center's vision on functional food definition and science in comparison to FDA's health claim authorization and Japan's Foods for Specified Health Uses. Functional Foods in Health and Disease 2020. 10(11), 465. DOI: https://doi.org/10.31989/ffhd.v10i11.753

9. Iwatani S, Yamamoto $\mathrm{N}$ : Functional food products in Japan: A review. FS and Human Wellness 2019. 8(2), 96101. DOI: https://doi.org/10.1016/j.fshw.2019.03.011

10. Consumers Affairs Agency [https://www.caa.go.jp/policies/policy/food labeling/h ealth_promotion/pdf/food labeling_cms206_200122_0 5.pdf] Retrieved June 25, 2021.

11. Martirosyan, D., Chen, S. FOSHU-approved Fibersol ${ }^{\circledR}-2$ product review. Bioactive Compounds in Health and Disease 2021. 4(5), 79. DOI: https://doi.org/10.31989/bchd.v4i5.797

12. Ono MA: Impacts of the FoSHU (Food for Specified Health Uses) system on food evaluations in Japan. Journal of Consumer Marketing. 2015, 32(7), 542-550. DOI: https://doi.org/10.1108/jcm-09-2014-1144

13. Martirosyan D., Miller E. Bioactive Compounds: The Key to Functional Foods. Bioactive Compounds in Health and Disease 2018; 1(3), 36-39. DOI: https://doi.org/10.31989/bchd.v1i3.539

14. Civil Eats. [https://civileats.com/2016/04/11/the-foodscience-lab-is-teaching-students-to-grow-food-in-achicago-high-school/] Retrieved July 25, 2021.

15. U.S. News and World Report-Education. [https://www.usnews.com/best-colleges/food-sciencemajor-0110] Retrieved July 25, 2021.

16. Martirosyan, D., Adany, A., Kanya, H. Japan's health food industry: An analysis of the efficacy of the FOSHU system. Bioactive Compounds in Health and Disease 2021. 4(4), 63. DOI: https://doi.org/10.31989/bchd.v4i4.795

17. 1Aggett, PJ: The process for the assessment of scientific support for claims on food. Eur J Nutr 2009. 48(23). DOI: https://doi.org/10.1007/s00394-009-0072-4

18. Alpha-Tocopherol, Beta Carotene Cancer Prevention Study Group. "The effect of vitamin E and beta carotene on the incidence of lung cancer and other cancers in male smokers." The New England Journal of Medicine.1994, 330(15), 1029-35. DOI: https://doi.org/10.1056/NEJM199404143301501

19. Crowe KM, Francis C: Position of the Academy of Nutrition and Dietetics: Functional Foods. Journal of the Academy of Nutrition and Dietetics 2013, 113(8), 10961103. DOI: https://doi.org/10.1016/j.jand.2013.06.002

20. Hecht SS: Chemoprevention of cancer by isothiocyanates, modifiers of carcinogen metabolism. The Journal of Nutrition. 1999, 129(3), 768S-774S. DOI: https://doi.org/10.1093/jn/129.3.768S.

21. Zhang $Y$, Talalay $P$ : Anticarcinogenic activities of organic isothiocyanates: chemistry and mechanisms. Cancer Res 1994. 54(7 Suppl), 1976s-1981s.
22. Getahun SM, Chung FL: Conversion of glucosinolates to isothiocyanates in humans after ingestion of cooked watercress. Cancer Epidemiol Biomarkers Prev. 1999. 8(5), 447-451.

23. Hecht SS: Inhibition of carcinogenesis by isothiocyanates*. Drug Metabolism Reviews 2000. 32(34), 395-411. DOI: https://doi.org/10.1081/DMR100102342.

24. Kris-Etherton, PM., Hecker, KD., Bonanome, A., Coval, SM., Binkoski, AE., Hilpert, KF., et all: Bioactive compounds in foods: their role in the prevention of cardiovascular disease and cancer. The American Journal of Medicine 2002. 113(9, Supplement 2), 71-88. DOI: https://doi.org/10.1016/S0002-9343(01)00995-0.

25. Teede, HJ., Dalais, FS., Kotsopoulos, D., Liang, Y-L., Davis, S., McGrath, BP:Dietary Soy Has Both Beneficial and Potentially Adverse Cardiovascular Effects: A PlaceboControlled Study in Men and Postmenopausal Women1. The Journal of Clinical Endocrinology and Metabolism. 2001, 86(7), 3053-3060. DOI: https://doi.org/10.1210/jcem.86.7.7645.

26. Tham, DM., Gardner, CD., Haskell, WL: Potential Health Benefits of Dietary Phytoestrogens: A Review of the Clinical, Epidemiological, and Mechanistic Evidence1. The Journal of Clinical Endocrinology and Metabolism. 1998, 83(7), 2223-2235. DOI: https://doi.org/10.1210/jcem.83.7.4752.

27. Kurzer, MS., Xu, X. Dietary Phytoestrogens. Annual Review of Nutrition 1997. 17(1), 353-381. DOI: https://doi.org/10.1146/annurev.nutr.17.1.353.

28. Setchell, KDR., Cassidy, A: Dietary Isoflavones: Biological Effects and Relevance to Human Health. The Journal of Nutrition. 1999, 129(3), 758S-767S. DOI: https://doi.org/10.1093/jn/129.3.758S.

29. Bingham, SA., Atkinson, C., Liggins, J., Bluck, L., Coward, A: Phyto-oestrogens: where are we now? British Journal of Nutrition. 1998, 79(5), 393-406. DOI: https://doi.org/10.1079/BJN19980068.

30. McMichael-Phillips, DF., Harding, C., Morton, M., Roberts, SA., Howell, A., Potten, CS: Effects of soy-protein supplementation on epithelial proliferation in the histologically normal human breast. The American Journal of Clinical Nutrition. 1998, 68(6), 1431S-1435S. DOI: https://doi.org/10.1093/ajcn/68.6.1431S.

31. Petrakis, NL., Barnes, S., King, EB., Lowenstein, J., Wiencke, J., Lee, MM., et all. Stimulatory influence of soy protein isolate on breast secretion in pre- and postmenopausal women. Cancer Epidemiol Biomarkers Prev. 1996. 5(10), 785-794.

32. Allred, CD., Allred, KF., Ju, YH., Virant, SM., Helferich, WG. Soy Diets Containing Varying Amounts of Genistein Stimulate Growth of Estrogen-dependent (MCF-7) Tumors in a Dose-dependent Manner. Cancer Res. 2001. 61(13), 5045-5050.

33. Dausch, JG., Nixon, DW. Garlic: A review of its relationship to malignant disease. Preventive Medicine 1999. 19(3), 346-361. DOI: https://doi.org/10.1016/0091-7435(90)90034-H.

34. Fukushima, S., Takada, N., Hori, T., Wanibuchi, H: Cancer prevention by organosulfur compounds from garlic and onion. Journal of Cellular Biochemistry. 1998, 67(S27). 
DOI:https://doi.org/10.1002/(SICI)10974644(1997)27+<100::AID-JCB16>3.0.CO;2-R

35. Crowell, PL: Prevention and Therapy of Cancer by Dietary Monoterpenes. The Journal of Nutrition. 1999, 129(3), 775S-778S. DOI: https://doi.org/10.1093/jn/129.3.775S.

36. Gould, MN. Prevention and therapy of mammary cancer by monoterpenes. Journal of Cellular Biochemistry. 1995, 59(S22), 139-144 DOI: https://doi.org/10.1002/jcb.240590818.

37. Elson, CE., Qureshi, AA. Coupling the cholesterol- and tumor-suppressive actions of palm oil to the impact of its minor constituents on 3-hydroxy-3-methylglutaryl coenzyme a reductase activity. Prostaglandins, Leukotrienes and Essential Fatty Acids 1995. 52(2), 205208. DOI: https://doi.org/10.1016/0952-3278(95)90024-1.

38. Ghould, MN., Moore, CJ., Zhang, R., Wang, B., Kennan, WS., Haag, JD. Limonene Chemoprevention of Mammary Carcinoma Induction following Direct in Situ Transfer of v-Ha-ras. Cancer Research 1994. 54(13), 3540-3543.

39. Löw-Baselli, A., Huber, WW., Käfer, M., Bukowska, K., Schulte-Hermann, R., Grasl-Kraupp, B. Failure to demonstrate chemoprevention by the monoterpene perillyl alcohol during early rat hepatocarcinogenesis: a cautionary note. Carcinogenesis 2000. 21(10), 18691877. DOI: https://doi.org/10.1093/carcin/21.10.1869.

40. Maruvada, P., Lampe, J.W., Wishart, D.S., Barupal, D., Chester, D.N., Dodd, D., et all. Perspective: Dietary Biomarkers of Intake and Exposure-Exploration with Omics Approaches. Advances in nutrition 2020. 11(2), 200-215. DOI:

https://doi.org/10.1093/advances/nmz075

41. Dragsted, L.O., Gao, Q., Scalbert, A., Vergères, G., Kolehmainen, M., Manach, C., et. all. Validation of biomarkers of food intake-critical assessment of candidate biomarkers. Genes and Nutrition 2018. 13(14). DOI: https://doi.org/10.1186/s12263-018-0603-9

42. Rafter, J. J. Scientific basis of biomarkers and benefits of functional foods for reduction of disease risk: cancer. British Journal of Nutrition. 2002, 88(S2), S219-S224. DOI: https://doi.org/10.1079/BJN2002686

43. Martirosyan D.M. The Emerging Potential of Functional Foods in Viral Disease Prevention. Functional Foods in Health and Disease 2020. 6(10), 95-99. DOI: https://www.doi.org/10.31989/bchd.v3i6.726

44. Howe, Peter RC. "What Makes a Functional Food Functional?" Asia Pacific J Clin Nutrition 2000. 9(S1), S108-S112. 